\title{
Feature Patch Illumination Spaces and Karcher Compression for Face Recognition via Grassmannians
}

\author{
Jen-Mei Chang1, Chris Peterson' ${ }^{2}$, Michael Kirby ${ }^{2}$ \\ ${ }^{1}$ Department of Mathematics and Statistics, California State University, Long Beach, USA \\ ${ }^{2}$ Department of Mathematics, Colorado State University, Fort Collins, USA \\ Email: jen-mei.chang@csulb.edu, \{peterson, Kirby\}@math.colostate.edu
}

Received January 7, 2012; revised February 20, 2012; accepted February 27, 2012

\begin{abstract}
Recent work has established that digital images of a human face, when collected with a fixed pose but under a variety of illumination conditions, possess discriminatory information that can be used in classification. In this paper we perform classification on Grassmannians to demonstrate that sufficient discriminatory information persists in feature patch (e.g., nose or eye patch) illumination spaces. We further employ the use of Karcher mean on the Grassmannians to demonstrate that this compressed representation can accelerate computations with relatively minor sacrifice on performance. The combination of these two ideas introduces a novel perspective in performing face recognition.
\end{abstract}

Keywords: Grassmannians; Karcher Mean; Face Recognition; Illumination Spaces; Compressions; Feature Patches; Principal Angles

\section{Introduction}

There has been a general philosophy in pattern recognition, arising out of practical necessity to some degree, to normalize away variations in imagery that appear nonessential to identification or classification. For example, in the context of human faces, recognition under uncontrolled illumination conditions has historically been one of the more difficult challenges. In an attempt to overcome this problem, several algorithms approximately remove illumination variations in an effort to improve recognition performance.

A number of papers have appeared recently which illustrate the potential of retaining effects of illumination variation over an object [1-4]. As a result, more generally, we are led to consider manners in which the variation in the state of an object can be used to extract discriminatory information. Philosophically, this paradigm shift encourages the collection and processing of large amounts of data to represent families of patterns. In this setting, the challenge now becomes how to encode and compare large quantities of information for pattern classification.

Because of the need for analyzing massive data sets, much recent effort has been devoted to developing pattern recognition methods that are based on image sets [5-8]. Typically, a signal or picture contains much redundant information that may be removed by using, e.g.,
Karhunen-Loève $(\mathrm{KL})$ transform. Each class then has its own set of representative features extracted from KL transform that forms a vector subspace (so-called feature space) of the original pattern space. The subspace method is a geometrically sound approach since these class subspaces can be used to classify an input sample into the best fitting class and they tell us something about the properties shared by all the items in that category. For this reason, subspace method works extremely well when samples are selected from a uniformly distributed variation state. Similarly, methods that are based on image sets give better performance than the ones based on individual images since classifiers that depend solely on a single input sample will be sensitive to outliers and anomalies.

Therefore, to improve and extend the traditional subspace method, we consider the case where both gallery and query subjects have multiple images available and refer this classification paradigm as the set-to-set method. Face recognition based on image set matching enjoys a superior discrimination accuracy and robustness since it is less sensitive to poor registration and varying environmental conditions. In many applications, such as video sequence matching, surveillance video tracking, spatiotemporal modeling, and affine invariant shape analysis, a set-to-set method provides the most natural platform for performing classification tasks. While there are multitude of ways to carry out the actual classification task, it has 
been shown that better performance can be attained through modeling image sets via linear structures, i.e., subspaces [5,7]. This is largely due to the positive effects inherited from the subspace method mentioned above. Next, we describe how classification on the Grassmann manifolds is an obvious choice for performing face recognition with linear structures.

The collection of multiple images for a single subject can be mathematically represented by a matrix of size $n$-by- $k$, where $k$ is the number of distinct patterns and $n$ is the resolution of the patterns. The linear span of the columns of this matrix forms a $k$-dimensional vector subspace in $\mathbb{R}^{n}$, which can be realized naturally as a point on the Grassmannian $G R(k, n)$. The detail of this connection is given in Section 2. Now, performing classification of sets (of patterns) in their natural setting is equivalent to performing classification of points on the Grassmannians. Distance measures on the Grassmannians are well-established in this context and can be applied readily to this problem. Overall, classification on the Grassmannians is a mathematically simple framework that can be extended to any pattern classification problem that requires a set-to-set data comparison. While there are other interesting general pattern analysis problems that fit naturally on the Grassmann manifold (see e.g., $[9,10]$, we focus on the ones that emphasize the use of principal angles, which are the fundamental building blocks of various unitarily invariant distance functions between linear subspaces [11].

As mentioned previously, it is evident that there is discriminatory information associated with the manner in which digital images of a face change under variations in illumination. Factors which affect these changes include the texture, color and shape of the face. One of the central themes of this paper is that the local information produced when illumination varies over sub-images of the face, e.g., facial features such as the eyes, lips or nose, will still allow accurate classification when placed in the context of set-to-set comparison via Grassmann manifolds. The extent to which local information is individualized is rather surprising and points to two immediate applications.

First, it suggests classification is possible even when a subject's face is severely occluded. It confirms with a lot of prior studies [12-15] that distinct features such as eyes, lip, and nose possess interesting information that can be used for classification. Second, it suggests that the information gained by considering multiple local illumination spaces may be substantially greater than the information gained from considering a single global illumination space. This second point differentiates our work from others. Precisely, our work is not to replicate the fact that local features preserve discriminatory structure; rather, the discriminatory structure of the whole face via illumination spaces persists through various ways of compression where the local feature patch being one of the many ways to select such reduced representation. Empirical results that validate this assertion are presented in Section 4. The classifier statistics was reported using a measure that is suitable on the Grassmann manifold and compared with a carefully chosen benchmark algorithm; both ideas are described in Section 2 as well.

One of the unavoidable consequences of the set-to-set paradigm is the increased classification runtime compared to single-to-single and single-to-many algorithms. One way to fix this is to replace the subject's image set with an invariant representation that captures the discriminatory variances afforded by the given set of images. Classification is then done on this invariant structure that is potentially much quicker to compute. To this end, we consider the notion of mean subspace on the Grassmann manifold. Similar to the idea of arithmetic mean in the Euclidean space, the mean on the Grassmann manifold minimizes the summed squared distance measured along the geodesics. Formally, this mean is called the Fréchet mean, or the Karcher mean if uniqueness criterion is required.

Collectively, this paper improves the face recognition problem with the original Grassmann (set-to-set) method in two aspects-by reducing the size of $k$ and $n$ in $G R(k, n)$. That is, by using the feature patches, we are essentially reducing the size of $n$; while by introducing the Karcher mean, we are essentially reducing the size of $k$. We give the details of achieving both types of compression in Section 3. The paper is concluded with Section 5 where a brief summary of the work presented will be given.

\section{The Grassmann Method}

The geometry of the data sets affects the fundamental design of a classification algorithm. For example, it is reasonable to quantify the distance between two points on the $x y$-plane embedded in $\mathbb{R}^{3}$ with Euclidean distance but the same metric should not be used to measure the distance between two points on the sphere. In any case, the optimal choice of the metric is the appropriate geodesic on that space. In this section, we review a geometric framework, so-called the Grassmann method, that is suitable for the set-to-set classification using linear subspaces for which the current research is built upon. The heart of our study centers around ways to improve the current Grassmann method by means of compression and will be described in Section 3.

\subsection{Matrix Representation for Points on the Grassmannians}

A $r$-by-c gray scale digital image corresponds to a $r \times c$ 
matrix where each entry enumerates one of the 256 (on 8-bit machines) possible gray levels of the corresponding pixel. After concatenation by columns, an image vector of length $n=r c$ can be seen as a point in $\mathbb{R}^{n}$ in the original subspace method, this point will then be projected into a feature space of a much lower dimension for classification. We will, however, group $k$ (generally independent) example images of a subject and consider the $k$-dimensional feature subspace they span in $\mathbb{R}^{n}$. The connection between this linear subspace to a point on the Grassmann manifold will be made precise next.

Definition 2.1 The Grassmannian $G R(k, n)$ or the Grassmann manifold is the set of k-dimensional suspaces in an $n$-dimensional vector space $K^{n}$ for some field $K$.

Let $K$ be a field and $V$ be a vector space of dimension $n$ with basis $\left\{e_{1}, e_{2}, \cdots, e_{n}\right\}$, it can be shown that the $k^{\text {th }}$ exterior power of $V$ over $K$ can be determined up to isomorphism. Then the Grassmannian, $G R(k, n)$, can be viewed as a subset of projective space, $\mathbb{P}\left(\wedge^{k} V\right)$, via the Plücker embedding:

$$
\begin{gathered}
G R(k, n) \stackrel{P l}{\longrightarrow} \mathbb{P}\left(\wedge^{k} V\right) \\
W \mapsto \wedge^{k} W
\end{gathered}
$$

where $\operatorname{dim}\left(\mathbb{P}\left(\wedge^{k} V\right)\right)=\left(\begin{array}{l}n \\ k\end{array}\right)-1$. This map is injective. The homogeneous coordinates on $\mathbb{P}\left(\wedge^{k} V\right)$ are called the Plücker coordinates on $G R(k, n)$ [16].

In coordinates, we can explicitly represent a plane $W \in G R(k, n)$ by a unique matrix up to a change of basis transformation. Let $W$ be a $k$-dimensional vector subspace of $V$ with basis $f_{j}=\sum_{i=1}^{n} b_{i j} e_{i}, j=1,2, \cdots k$ and let $B=\left[b_{i j}\right]$. Moreover, assume $U$ is the standard affine open subset of $\mathbb{P}\left(\wedge^{k} V\right)$ whose first $k \times k$ minor is nonzero. Then

$$
B=\left[b_{i j}\right] \sim\left[\begin{array}{c}
I_{k} \\
B_{(n-k) \times k}^{\prime}
\end{array}\right] .
$$

The matrix $B$ is determined up to right multiplication by an invertible $k \times k$ change of basis matrix. $B$ uniquely determines $B^{\prime}$, and $B^{\prime}$ uniquely determines $W$. Then the entries of $B^{\prime}$ give the bijection of $U \cap G R(k, n)$ with $K^{k(n-k)}$, i.e., $G R(k, n)$ is covered by affine space of dimension $k(n-k)$. Consequently, $\operatorname{dim}(G R(k, n))=$ $k(n-k)$ when the Grassmannian is realized as a submanifold of a projective space.

It is now clear that points in the Grassmannian are equivalence classes of $n \times k$ orthonormal matrices, where two matrices are equivalent if their columns span the same $k$-dimensional linear subspace, i.e.,

$$
G R(k, n)=\left\{[p]: p \sim q \text { if } f q=Q^{T} p \text { for some } Q \in O_{k}\right\}
$$

where $p$ and $q$ are $n \times k$ matrices with orthogonal col- umns and $O_{k}$ is the group of $k \times k$ orthogonal matrices.

Therefore, the Grassmann manifold $G R(k, n)$ can be identified as the quotient group $O_{n} /\left(O_{k} \times O_{n-k}\right)$. Despite this abstract mathematical representation of the

Grassmannian, one may choose to represent a point on the Grassmannian by specifying an arbitrary orthonormal basis stored as a $n \times k$ matrix. Although this choice of the orthogonal matrix is not unique for points on the Grassmannian, it does give rise to a $k$-dimensional linear subspace that is obtained via the column space of the matrix and will serve as are presentative of the equivalence class on the computer [17].

\subsection{Geometry of the Grassmannians}

A natural question that follows is the way in which we measure how far apart points are on the Grassmann manifold. In the context of face recognition, by realizing sets of images as points on a Grassmann manifold, we can take advantage of the geometries imposed by individual metrics (drawn from a large class of metrics) in comparing the closeness of the points. For instance, the arc length metric and the Fubini-Study metric impose quite distinct geometries on the Grassmann manifold and lead to distinct distance measures between points. It is an open question how to optimally select a metric on a Grassmannian for the purposes of a given data classification problem. See [17] for a list of several commonly used metrics. We will restrict ourselves to metrics on $G R(k, n)$ built as functions of the $k$-tuple of principal angles. For instance, in the arc length metric, the distance between $p, q \in G R(k, n)$ is written in terms of the principal angles $\theta(p, q)=\left(\theta_{1}, \theta_{2}, \cdots, \theta_{k}\right)$ as

$$
d_{g}(p, q)=\left(\sum_{i=1}^{k} \theta_{i}^{2}\right)^{1 / 2}
$$

In the following paragraphs, we review the definition of principal angles and an algorithm for computing them.

If $\mathcal{X}$ and $\mathcal{Y}$ are two vector subspaces with $\mathcal{X}=\operatorname{span}(X)=\mathcal{R}(X)$ and $\mathcal{Y}=\operatorname{span}(Y)=\mathcal{R}(Y)$, where $\mathrm{X} \in \mathbb{R}^{n \times p}$ and $\mathrm{Y} \in \mathbb{R}^{n \times q}$ are two orthonormal basis matrices, then the principal angles $0 \leq \theta_{k} \leq \pi / 2$,

$1 \leq k \leq q=\min \{\operatorname{dim}(X), \operatorname{dim}(Y)\}$, between $\mathcal{X}$ and $\mathcal{Y}$ are defined recursively by

$$
\cos \theta_{k}=\max _{u \in \mathcal{X}} \max _{v \in \mathcal{Y}} u^{T} v=u_{k}^{T} v_{k}
$$

subject to $\|u\|_{2}=\|v\|_{2}=1, \quad u^{T} u_{i}=0$ and $v^{T} v_{i}=0$ for $i=1,2, \cdots, k-1$. That is, the first principal angle, $\theta_{1}$, is the smallest angle between all possible linear combinations of unit vectors in $\mathcal{X}$ and $\mathcal{Y}$ and the second principal angle, $\theta_{2}$, is the smallest angle between the spaces $\left(\mathcal{X}-u_{1}\right)^{\perp}$ and $\left(\mathcal{Y}-v_{1}\right)^{\perp}$, and so on. The vectors $u_{i}$ and $v_{i}$ are so-called the $i^{\text {th }}$ left and right principal vectors corresponding to the $i^{\text {th }}$ principal angle. The use of principal 
vectors and principal angles help us in answering the following question: "what linear combination of images in one set comes closest to a linear combination of images in the second set?" If we name the sets "left" and "right", we can then describe the closest pair of linear combinations of images as the left and right principal vectors.

A numerically stable algorithm that computes the principal angles between subspaces $\mathcal{X}$ and $\mathcal{Y}$ is given in [18]. This algorithm is accurate for large principal angles $\left(>10^{-8}\right)$. A sine-based algorithm for calculating small principal angles is available in [19]. This algorithm, presented in Algorithm 1, is used in the present paper to ensure precision of the minimal principal angles. Given the nature of the data investigated in this paper, we found it sufficient to consider only the minimum principal angle in measuring the similarity between two points on a Grassmannian generated by two image sets, see e.g., [20].

We note that face recognition using principal angles not in the context of the geometry of Grassmann manifolds-can be traced back to the Mutual Subspace Methodwhere the cosine of the minimal principal angle is used [5]. Since then, the concept of principal angles as a measure has been widely adopted [6,21-24], though still not as a way of metric on the Grassmann manifold until recently. Kernel methods for handling nonlinearity in data in the context of Grassmannian has been proposed as an extension of [4] and [25] and shown to be successful

Algorithm 1. Small and large principal angles [19].

Inputs: Matrices $X(n \times p)$ and $Y(n \times p), q \leq p$.

Outputs: Principal angles $\theta=\left[\theta_{k}\right]$ between $\mathcal{R}(X)$ and $\mathcal{R}(Y)$.

1) Find ONB and $Q_{x}$ for $Q_{y} \mathcal{R}(X)$ and $\mathcal{R}(Y)$ such that

$$
Q_{x}^{T} Q_{x}=Q_{y}^{T} Q_{y}=I, \mathcal{R}\left(Q_{x}\right)=\mathcal{R}(X), \mathcal{R}\left(Q_{y}\right)=\mathcal{R}(Y) .
$$

2) Compute SVD for cosine:

$$
Q_{x}^{T} Q_{y}=H \Sigma Z^{T},
$$

where $\Sigma=\operatorname{diag}\left(\sigma_{i}\right)$.

3) Compute matrix

$$
Y= \begin{cases}Q_{y}-Q_{x}\left(Q_{x}^{T} Q_{y}\right), & \text { if } \operatorname{rank}\left(Q_{x}\right) \geq \operatorname{rank}\left(Q_{y}\right) \\ Q_{x}-Q_{y}\left(Q_{y}^{T} Q_{x}\right), & \text { otherwise. }\end{cases}
$$

4) Compute SVD for sine:

$$
\left[H, \operatorname{diag}\left(u_{i}\right), Z\right]=\operatorname{svd}(Y),
$$

5) Compute the principal angles, for $k=1,2, \cdots, q$ :

$$
\theta_{k}= \begin{cases}\arccos \left(\sigma_{k}\right), & \text { if } \sigma_{k}^{2}<\frac{1}{2} \\ \arcsin \left(\mu_{k}\right), & \text { if } \mu_{k}^{2} \leq \frac{1}{2}\end{cases}
$$

[7,26-28]. Readers who are interested in how the Grassmann method compares against other existing set-based and non-set-based methods in both classifier accuracy and computational cost are referred to [7,28,29]. In summary, non-set-based methods are outperformed by set-based ones which are now shadowed by the rapid development of kernel methods in the Grassmannian framework. An extensive review of these methods is beyond the scope of this paper.

\subsection{Classification on the Grassmannians}

Suppose $k$ distinct images of a given subject are available, we group them to form a data matrix $X$ with each image stored as a column of $X$. If the column space of $X$, $\mathcal{R}(X)$, has dimension $k$ and if $n$ denotes the image resolution, then $\mathcal{R}(X)$ is a $k$-dimensional vector subspace of $\mathbb{R}^{n}$, which corresponds to a point on the Grassmann manifold $G R(k, n)$. Under this framework, each set of $k$ images may be encoded as a point on the Grassmann manifold. There are various ways to perform classification on the Grassmann manifold. Since the heart of this analysis is to illustrate the discriminatory information inherited in the subject feature patches when viewed under varying illumination conditions, the only intrinsic variation presented in each image set is the variation of illumination.

As illustrated in Figure 1, images from each image set belong to a particular subject class and capture a given feature patch that is pre-defined succeeding the classification. Note that the illumination conditions do not need to be the same across image sets and the number of principal angles available between each pair of image sets depends on the size of the sets. In this simplistic 2-class classification problem, the probe set (image set 3 ) is closer to image set 1 than image set 2 , therefore is classified as subject 1 . On the other hand, it is possible to include two or more states of variations in the image sets;

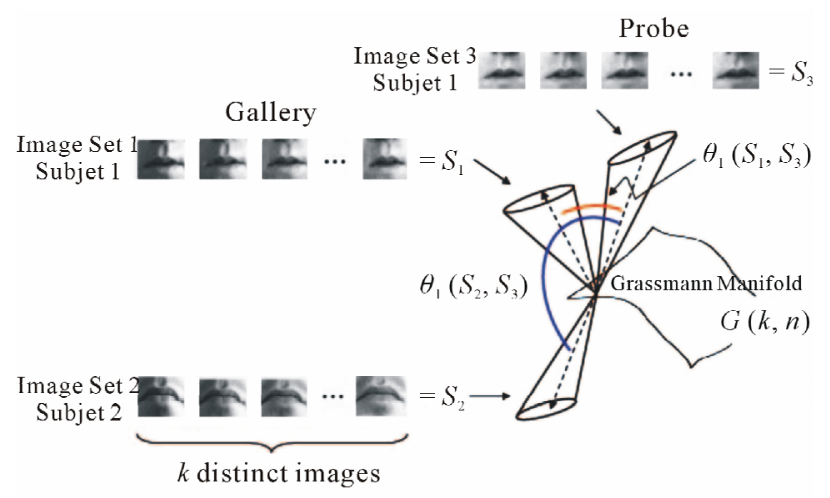

Figure 1. Sets of images are realized as points on the Grassmann manifold. The closeness of each pair of image sets may be measured, e.g., by the minimal principal angle, $\theta_{1}$, between the linear span of the image sets. 
however, it will become increasingly more difficult to identify the source of discriminatory information, and this problem is not considered in the current study.

\subsection{Computational Comlexity}

Classification on the Grassmann manifold comprises two major computational steps: an orthogonal basis extraction and a pair wise angle calculation. The algorithm for computing principal angles between a pair of subspaces also consists of two major steps: a QR-decomposition of the representation matrices and SVD of the inner product of the orthogonal matrices. The MATLAB qr command is based on Householder reflections. For a general $n \times k$ matrix representation for a point on $G R(k, n)$, QR-decomposition using Householder reflections costs

$2 k^{2} n-\frac{2}{3} k^{3}$ flops. For the same size matrix, the MATLAB svd command costs $4 k^{2}\left(n-\frac{1}{3} k\right)$ flops to reduce it to a bidiagonal form using Householder reflections. If singular values are required, it costs $O\left(k^{2}\right)$ for the rest of the operations.

Typically, $n \gg k$ and since the SVD is performed on the $k \times k$ covariance matrix, the overall computational cost for calculating the angles between a pair of points on $G R(k, n)$ is given by

$$
C(k, n)=2 n k^{2}+2 k^{3}+O\left(k^{2}\right) .
$$

Notice that this cost function is linear in $n$, the image resolution, and cubic in $k$, the number of images.

\subsection{Grassmann Separability}

Since this paper is about matching one set of face images to another set of face images, the common terms gallery and probe set are altered here to describe sets of sets of images. Thus, we consider the gallery data to consist of a set of points on a Grassmann manifold where eachpoint in the gallery is generated by computing a basis from a set of images associated with a given person. Points are computed in a similar fashion for the probe. Further, we assume that we know the labels of the points in both the probe and gallery permitting us to evaluate classification accuracy. In this section, we will introduce a quantity that measures the classifying power of the proposed framework that is appropriate on the Grassmann manifold.

Given a set of image sets $\mathcal{P}=\left\{X_{1}, X_{2}, \cdots, X_{m}\right\}$ and an identify map $\phi$ so that $\phi\left(X_{i}\right)=c_{i}$ where

$c_{i} \in\{1,2, \cdots, N\}$ is a set of class labels. Let the cardinality of a set, $X$, be the number of distinct images in $X$. The distances between different realizations of subspaces for the same class are called match distances while for dif- ferent classes they are called non-match distances. For simplicity of notations, define $W_{i}=\left\{j \mid \phi\left(X_{j}\right)=i\right\}$, the within-class index set of subject $i$ and $B_{i}=\{j \mid \phi \neq i\}$, the between-class index set of subject $i$. We can now define a quantity that measures how separable the data set is.

Definition 2.2. For an appropriate choice of the metric, $d$, let $M$ be the maximum of the match distances, i.e.,

$$
M=\max _{1 \leq i \leq m} \max _{j \in W_{i}} d\left(X_{i}, X_{j}\right)
$$

and $m$ be the minimum of the non-match distances, i.e.,

$$
m=\min _{1 \leq i \leq m} \min _{k \in B_{i}} d\left(X_{i}, X_{k}\right) .
$$

Define the separation gap as $g_{s}=m-M$. Then we say the set $\mathcal{P}=\left\{X_{1}, X_{2}, \cdots, X_{m}\right\}$ is Grassmann separable if $g_{s}>0$.

There are two parameters in this definition, i.e., the choice of the metric and the configuration of the image sets. In this study, we speak of a set being Grassmann separable if there exists a Grassmannian distance and a set configuration such that the separation gap is greater than zero. Pictorially, if we compile all of the withinclass and between-class distances of a Grassmann separable set, its box-whisker plot would resemble something similar to Figure 2(a) while a set that is not Grassmann separable would produce a box-whisker plot similar to that of Figure 2(b).

The Grassmann separability defined such way coincides nicely with a classifier metric termed false accept rate (FAR) at a zero false reject rate $(\mathrm{FRR})^{1}$ that is documented in [30]. This score is the ratio of the number of non-match distances that are smaller than the maximum of the match distances divided by the number of nonmatch distances. Essentially, the FAR score is capturing the separation gap described earlier and a zero FAR for a data set indicates that the data is Grassmann separable for an appropriate choice of metric and set configuration. Henceforth, the FAR score will be reported throughout the experiments as a way to tell how well proposed framework works. Moreover, due to the nature of the Grassmann separability criterion, a careful examination on how the cardinality of the image sets affect FAR scores will also be investigated in the experiments.

\subsection{Benchmark Algorithm}

We are sensitive to the concern that similar outcomes might be observed using direct image set to image set comparisons. To explore this, we introduce a benchmark similarity $S(X, Y)$ for comparing multi-still sets $X$ and $Y$ that exploits only the statistics of the image sets without imposing any geometric structure on the data. Since the

\footnotetext{
${ }^{1}$ For simplicity, we will use FAR to denote FAR at zero FRR.
} 


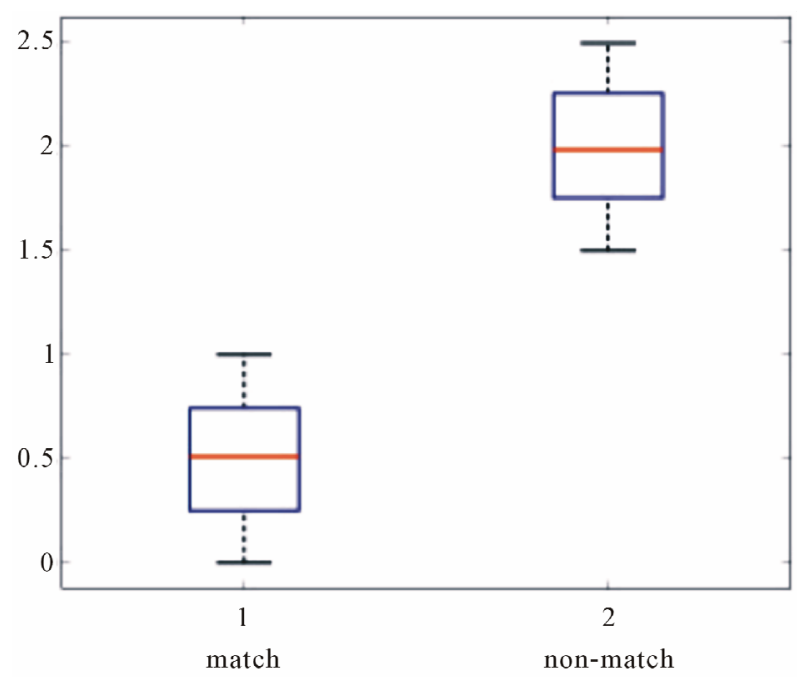

(a)

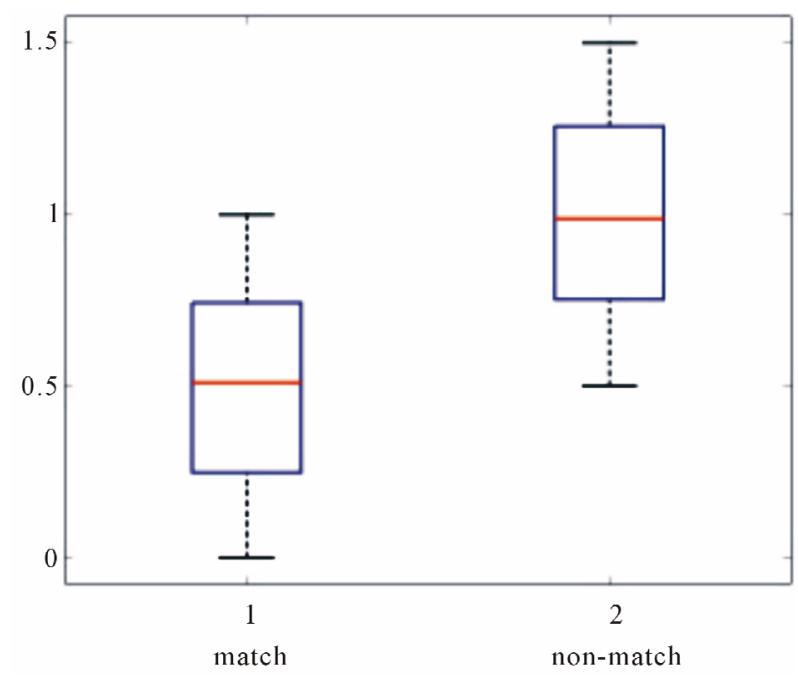

(b)

Figure 2. (a) Illustration of a data set that is Grassmann separable; (b) Illustration of a data set that is not Grassmann separable.

emphasis of the current study is to examine whether the discriminatory nature of the points on the Grassmann manifold persists through various compression schemes, we use only this algorithm to benchmark the performance of the proposed framework. Largely due to the nature of the methods and experimental protocols implemented, a direct comparison of our proposed framework with general face recognition techniques is difficult without a careful paradigm design.

Recall the familiar Pearson's $r$-correlation for two length $N$ column vectors $x$ and $y$ :

$$
\operatorname{Cor}(x, y)=\frac{1}{N-1} \sum_{j=1}^{N} \frac{x_{j}-\bar{x}}{\sigma_{x}} \times \frac{y_{j}-\bar{x}}{\sigma_{y}},
$$

where $\sigma_{x}$ and $\sigma_{y}$ are the standard deviations and $\bar{x}$ and $\bar{y}$ are the means of the signals $x$ and $y$, respectively. Now, for two image sets

$$
X=\left[x^{(1)}\left|x^{(2)}\right| \cdots \mid x^{\left(k_{x}\right)}\right]
$$

and

$$
Y=\left[y^{(1)}\left|y^{(2)}\right| \cdots \mid y^{\left(k_{y}\right)}\right]
$$

set

$$
s\left(x^{(j)}, Y\right) \max _{1 \leq i \leq k_{y}}\left\{\operatorname{Cor}\left(x^{(j)}, y^{(i)}\right)\right\}
$$

to be the similarity score between a single image $x^{(j)}$ in $X$ and the image set $Y$. Then our benchmark similarity between $X$ and $Y$ is defined as

$$
S(X, Y)=\frac{1}{2} \sum_{i=1}^{k_{X}} \sum_{j=1}^{k_{y}}\left(s\left(x^{(i)}, Y\right)+s\left(y^{(j)}, X\right)\right) .
$$

This definition permits a symmetric measure and is essentially an exhaustive approach in searching for the best match in image sets.

\section{Compressions on the Grassmannians}

Let $S$ be a collection of points on a Grassmann manifold $G R(k, n)$ with each point corresponding to a set of $k$ digital images each residing in $\mathbb{R}^{n 2}$. We will consider two types of compressions of such data that still allow classification. The first type of compression has the effect of reducing $n$ while the other reduces $k$. Both types of compression yield new collections of points on Grassmann manifolds. A compression which reduces $n$ corresponds to reducing the number of pixels representing a digital image. Reduction in the size of $k$ corresponds to reducing the dimension of the subspace representing a set of digital images. There might be other methods for accomplishing either of these tasks while we will consider reductions in $n$ induced by projections in Section 3.1 and reductions in $k$ through a Karcher mean computation in Section 3.2.

\subsection{Compression of $\boldsymbol{n}$ in $G R(k, n)$}

As demonstrated in [2], the illumination space of a Lambertian object is well approximated by a low-dimensional linear space. This implies that if $D$ represents a data set consisting of digital images of a fixed Lambertian object collected under a variety of illumination conditions and with a fixed resolution, then a very high percentage of the energy of $D$ is captured by a low-dimensional linear space inside the vector space generated by all possible digital images at the same fixed resolution. As a consequence, illumination spaces are particularly well-suited

${ }^{2}$ It is not necessary to perform a preliminary reduction of the data using a method such as the SVD. 
for classification on Grassmannians.

The approximated illumination space, as captured by a $k$-dimensional approximation of the data set $D$, can be represented by a point $p \in G R(k, n)$. Given a point $q \in G R(k, n)$, let $V_{q} \subset \mathbb{R}^{n}$ denote the associated $k$-dimensional subspace. In typical settings, where $n, m$ are much larger than $k$, one expects a general linear map $L: \mathbb{R}^{n} \rightarrow \mathbb{R}^{m}$ to approximately preserve the inter-relationships among the principal angles between most subspaces of $\mathbb{R}^{n}$. For instance, suppose $V_{p}, V_{q}, V_{r}$ are three $k$-dimensional subspaces of $\mathbb{R}^{n}$ (corresponding to three points $p, q, r \in G R(k, n))$, suppose further that the minimal principal angle between $V_{p}$ and $V_{q}$ is small compared to the minimal principal angle between $V_{p}$ and $V_{r}$. Then under the general linear map $L$, one expects the minimal principal angle between $L\left(V_{p}\right)$ and $L\left(V_{q}\right)$ to be small compared to the minimal principal angle between $L\left(V_{p}\right)$ and $L\left(V_{r}\right)$.

There are several families of linear transformations which are natural and useful to consider in the context of face recognition. In this paper we will restrict our attention to a special family of linear transformations known as patch projections, these should be considered as complementary to patch collapsing. These terms are described below:

1) (Patch Collapsing) Consider a partition of the components of a vector, $V$, into disjoint sets $P_{1} \cup P_{2} \cup \cdots \cup P_{d}$. Patch collapsing is the operation of replacing, for each $i$ between 1 and $d$, the components in $P_{i}$ with a fixed weighted average of these components. This operation can be expressed as a linear map, $L: \mathbb{R}^{n} \rightarrow \mathbb{R}^{d} \subset \mathbb{R}^{n}$, $d \ll n$. If $L$ is further required to conserve energy then $L \circ L=L$ and thus is a projection map. An example of this type of projection is the partitioning of a digital image into regions as provided by the scaling spaces in the Haar wavelet decomposition. See Figure 3(a) for an illustration of this type.

2) (Patch Projection) Given a partition of the components of a vector, $V$, into disjoint sets $P_{1} \cup P_{2} \cup \cdots \cup P_{d}$. A family of patch projections is given by the natural projection maps $L_{i}: \mathbb{R}^{n} \rightarrow \mathbb{R}^{\left|P_{i}\right|},\left|P_{i}\right| \ll n$. An example of a patch projection is the restriction of a digital image to a region of the image. For instance, the restriction of a digital image of a face to the region surrounding the lips. See Figure 3(b) for illustrations of several patch projections.

Patch projection is the focus of this paper, where a patch of an image is simply a sub-image with resolution much less than the original full image. Patch projections are linear maps and illumination spaces are well approximated by linear spaces thus it is natural to study patch illumination spaces through the mathematics associated to parameter spaces of linear spaces. A few comments on the relationship between projections and Grass- mannians are in order.

Let $K$ be the kernel of a linear map $L: \mathbb{R}^{n} \rightarrow \mathbb{R}^{m}$. Let $\bar{\Omega}(K) \subset G R(k, n)$ denote the Schubert variety defined by

$$
\bar{\Omega}(K)=\left\{p \in G R(k, n) \mid \operatorname{dim}\left(V_{p} \cap K\right) \geq 1\right\} .
$$

$L$ induces a natural map

$$
L_{G}: G R(k, n)-\bar{\Omega}(K) \rightarrow G R(k, m)
$$

since the image of any $k$-dimensional subspace $V \subset \mathbb{R}^{n}$ under $L$ remains $k$-dimensional precisely if the point $p \in G R(k, n)$ corresponding to $V$ lies outside of $\bar{\Omega}(K)$. Suppose $\operatorname{dim} K+k \leq n$, then $\bar{\Omega}(K)$ is a proper subset of $G R(k, n)$ and the dimension of $\bar{\Omega}(K)$ is strictly less than the dimension of $G R(k, n)$. Thus, with probability one, a point chosen at random from $G R(k, n)$ will lie in $G R(k, n)-\bar{\Omega}(K)$. Due to the method we use to determine points on $G R(k, n)$, the quantization of pixel values in digital images and the special nature of patch projections, we are not choosing random points. Thus it is possible for the corresponding linear spaces to have a non-trivial intersection with the kernel of the projection map. However, as one might expect, we have yet to observe a point accidently chosen to lie within $\bar{\Omega}(K)$.

The computational saving that is accomplished by this type of compression is on the order of $O(n)$. For example, using image patches of $30 \times 30$ instead of the original $200 \times 200$ will enable a speedup that is roughly 44 times faster for a single pair wise distance calculation.

\subsection{Compression of $k$ in $G R(k, n)$}

The notion of mean is often used as an initial estimator for studying variability in a distribution. We anticipate the use of mean subspaces, in object recognition problems that are cast on a Grassmannian, will provide us a
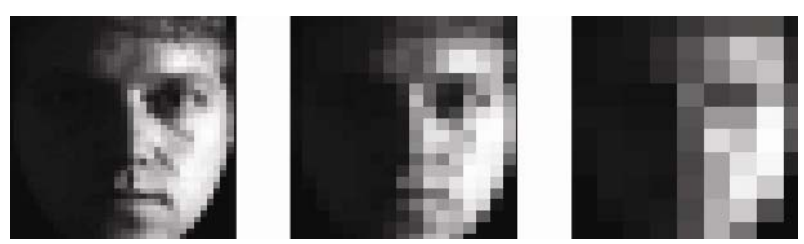

(a)
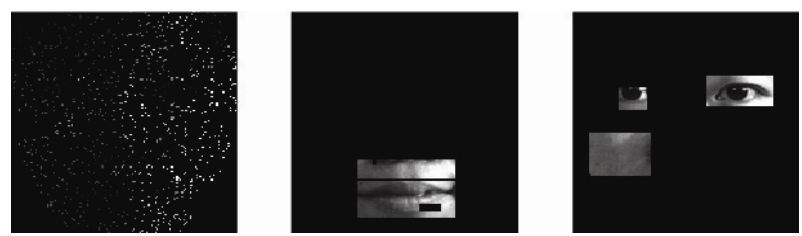

(b)

Figure 3. (a) An example of patch collapsing provided by the scaling spaces in the Haar wavelet decomposition; (b) Illustration of patch projections. Patches do not have to be selected from a connected nor a rectangular region. 
blueprint to embed discriminatory information through spaces of reduced dimensions. In this section, a quick overview for calculating the mean subspace on $\operatorname{GR}(k, n)$ will be given followed by a proposed algorithm for performing a robust classification at reduced computational cost. We emphasize that it is the machinery associated with the Grassmann manifold that permits this construction.

Although the definition of the Karcher mean is wellestablished and it is easy to implement an algorithm for its effective computation (see, e.g., [31,32]), the calculation of a Karcher mean can be rather expensive. Even on a relatively small collection of sets, the computation can fail to finish in a satisfactorily short period of time.By incorporating low resolution feature patches in construction of the subject illumination spaces, as suggested in this paper, the algorithm for calculating the Karcher mean becomes computationally tractable. We now briefly review the essential notations and algorithms for calculating the Karcher mean for collections of points on the Grassmann manifold.

Given points $p_{1}, \cdots, p_{m} \in G R(k, n)$, the Karcher mean is the point $q^{*}$ that minimizes the sum of the geodesic distances between $q^{*}$ and the $p_{i}$ 's, i.e.,

$$
q^{*}=\underset{q \in G R(k, n)}{\operatorname{argmin}} \frac{1}{2 m} \sum_{j=1}^{m} d^{2}\left(q, p_{j}\right),
$$

where $d(p, q)$ is the geodesic distance between $p$ and $q$ on the Grassmannian. We adopt a SVD-based algorithm for computing the Karcher mean on a Grassmann manifold as given in [33], which will be reviewed next.

Recall a point $p \in G R(k, n)$ corresponds to a $k$-dimensional subspace of $\mathbb{R}^{n}$ and can be represented by a $n \times k$ matrix with orthonormal columns. Two matrices with orthonormal columns, $M, N$ determine the same point in $G R(k, n)$ if and only if $M=N Q$ for some $\in O_{k}$, where $O_{k}$ is the orthogonal group of $k \times k$ matrices. The tangent space $T_{p} G R(k, n)$ to $p \in G R(k, n)$ is given by

$$
T_{p} G R(k, n)=\left\{w \mid w=p_{\perp} q,\left\{w \mid w=p_{\perp} q, p_{\perp}=N\left(p^{T}\right)\right\},\right.
$$

where $g \in \mathbb{R}^{(n-k) \times n}$. Notice that $p_{\perp}$ is the orthogonal compliment of $p$. The $\operatorname{EXP}_{p}$ map that takes a point in the tangent space $T_{p} G R(k, n)$ to a point in $G R(k, n)$ is given by

$$
\operatorname{Exp}_{p}: T_{p} G R(k, n) \rightarrow G R(k, n)
$$

where

$$
w \in T_{p} G R(k, n) \rightarrow p V \cos \Theta+U \sin \Theta
$$

hasthe $\mathrm{SVD} w=U \Theta V^{T}$. The $\log _{p}$ map that takes a point in a neighborhood of $p \in G R(k, n)$ to a point in $T_{p} G R(k, n)$ is given by

$$
\log _{p}: q \in U_{p} \subset G R(k, n) \rightarrow T_{p} G R(k, n)
$$

with $q \rightarrow U \Theta V^{T}$, where $p_{\perp} p_{\perp}^{T} q\left(p^{T} q\right)^{-1}=U \Sigma V^{T}$ and $\Theta=\arctan \Sigma$, when it is well-defined. A descent method that utilizes the Exp and Log maps for finding the Karcher mean on the Grassmann manifold is given in Algorithm 2. For convergence results, see [33]. Next, we describe an novel algorithm that utilizes Karcher mean on $G R(k, n)$ to construct a compressed representation for a given collection of images that captures the intrinsic variability of the subject illumination space.

Given a set of $N$ images for a fixed subject class and prescribe a Karcher representation dimension $k$, repeat the following two steps for a total of $t$ times, where $t$ is a number greater than 1 and usually much less than $\left(\begin{array}{l}n \\ 2\end{array}\right)$. The purpose of the repetition is to capture the set variability exhibited in data set.

1) Randomly split the available data into two disjoint setsof equal size each containing $\left\lfloor\frac{N}{2}\right\rfloor$ images and the data into so that $\mathcal{R}\left(T_{m}\right)$ and $\mathcal{R}\left(Q_{m}\right)$ are two points on $G R\left(\left\lfloor\frac{N}{2}\right\rfloor, n\right)$, where $n$ is the resolution of the images.

2) Compute the first $k$ left principal vectors of the pair of subspaces $\mathcal{R}\left(T_{m}\right)$ and $\mathcal{R}\left(Q_{m}\right)$ and store the resulting vectors in an $n \times k$ matrix, $l_{m}$.

The collection of $t$ principal vectors, $\left\{l_{m}\right\}_{1 \leq m \leq t}$, corresponds to $t$ points on $G R(k, n)$. A $k$-dimensional, $1 \leq k \leq\left\lfloor\frac{N}{2}\right\rfloor$, compressed representation of the illumination feature patch space for the subject class is then given by the Karcher mean of the set $\left\{l_{m}\right\}_{1 \leq m \leq t}$ via Algorithm 2 and is denoted by $\langle l\rangle_{K}$. A detailed description of the algorithm is given in Algorithm 3 with a schematic illustration given in Figure 4.

Although Algorithm 3 is expensive even with the low-resolution patch images due to the number of singular value decomposition it requires, the actual computations are done off-line. Moreover, each subject's $k$-dimensional Karcher representation, with $k$ much less than

\section{Algorithm 2. Karcher mean on $G R(k, n)$ [33,34].}

Inputs: Points $p_{1}, p_{2}, \cdots, p_{m} \in G R(k, n), \epsilon$ (machine precision). Outputs: Karcher mean, $q$, of $\left\{p_{1}, p_{2}, \cdots, p_{m}\right\}$.

1) Set $q=p_{1}$.

2) Find $A=\frac{1}{m} \sum_{i=1}^{m} \log _{q}\left(p_{i}\right)$.

3) If $\|A\|<\epsilon$, return $q$; else, go to step 4 .

4) Find the SVD: $U \Sigma V^{T}=A$. Update $q \rightarrow q V \cos \Sigma+U \sin \Sigma$, go to step 2 . 
Algorithm 3. Karcher representation.

Inputs: $k$ (Karcher dimension), $t$ (training iteration), $N$ images for a fixed subject class.

Outputs: $k$-dimensional subject Karcher representation, $\langle l\rangle_{K}$.

1) For each training iteration $m=1: t$, do the following:

a) Let $T_{m}$ and $Q_{m}$ be two matrices such that $T_{m}, Q_{m} \in \mathbb{R}^{n \times\left\lfloor\frac{N}{2}\right\rfloor}$ and $\mathcal{R}\left(T_{m}\right)$ and $\mathcal{R}\left(Q_{m}\right)$ do not intersect trivially. Columns of $T_{m}$ and $Q_{m}$ are selected from the $N$ input images.

b) Find the first $k$ left principal vectors of the pair of subspaces $\mathcal{R}\left(T_{m}\right)$ and $\mathcal{R}\left(Q_{m}\right)$ :

$$
\begin{gathered}
T_{m}=Q_{t} R_{t}, Q_{t}^{T} Q_{t}=I, R_{t} \in \mathbb{R}^{\left\lfloor\frac{N}{2}\right\rfloor\left\lfloor\frac{N}{2}\right\rfloor}, \\
Q_{m}=Q_{q} R_{q}, Q_{q}^{T} Q_{q}=I, R_{q} \in \mathbb{R}^{\left\lfloor\frac{N}{2}\right\rfloor\left\lfloor\frac{N}{2}\right\rfloor}, \\
M=Q_{t}^{T} Q_{q} .
\end{gathered}
$$

Compute the SVD of $M=Y S Z^{T}$. The left principal vectors are given by columns of $U=Q_{t} Y$. Let the first $k$ left principal vectors be $l_{m}=U(:, 1: k)$.

2) Find the Karcher mean of $\left\{l_{m}\right\}_{1 \leq m \leq t},\langle l\rangle_{K}$, with Algorithm 2.

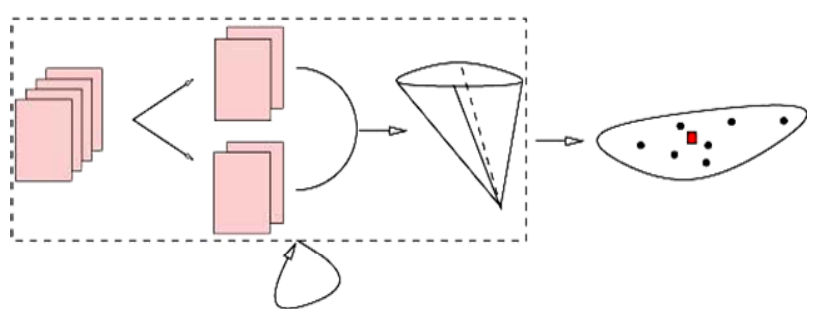

Figure 4. A schematic illustration for Algorithm 3. The boxed step is repeated $t$ times to create $t$ points on $G R(k, n)$.

The square element is then the Karcher mean of the circle points on $G R(k, n)$.

the total number of images available, captures the most significant discriminatory information and takes much less space to store on a machine. This way, we can use a single $k$-dimensional subspace to represent a subject class in the gallery, hence using less storage space while speeding up online classification runtime.

The computational saving that is accomplished by this type of compression is on the order of $O\left(k^{3}\right)$. For example, using a 3-dimensional Karcher representation instead of the original 12-dimensional subject subspace representation will enable a speedup that is roughly 64 times faster for a single pair wise distance calculation.

\section{Experiments and Results}

In this section, some proof-of-concepts experiments are designed to demonstrate that the idiosyncratic nature of subject illumination subspaces persist through two types of compression on the Grassmannians, $G R(k, n)$. In the first set of experiments, the value of $k$ is fixed to ten while $n$ is given by the resolution of the corresponding feature patch which translates to compression in $n$. In the second set of experiments, we explore how the performance of the classifier changes as we vary values of $k$, hence corresponding to compression in $k$. Since the Grassmann method does not require a training phase, we use the common terms "gallery" and "probe" to simply mean two sets of images used in the classification process.

\subsection{Data Sets}

The first data set we use to empirically test the performance of feature patches in a face recognition problem are the "illum" and "lights" subsets of the CMU-PIE Database [35], see Figure 5 for an illustration of the illumination variations on the selected nose patch for a fixed per$\operatorname{son}^{3}$. The images are normalized according to known eye coordinates. The viewpoint is fixed to be frontal and subsets of 21 distinct illumination conditions are used to form the probe and gallery.

The second data set we consider is a private face database, CSU-PAL, collected in the Pattern Analysis Lab (PAL) at Colorado State University (CSU). The purpose of introducing this database is to demonstrate the practicality of the proposed framework given a reliable eye detector is in place.

The current database (continuously expanding) contains face images of 100 subjects under three different lighting conditions (illumination variation with ambient lights on, illumination variation with ambient lights off, and no illumination variation with ambient lights on) and ten distinct protocols (still neutral expression, smile, frown, angry, puzzled, count to ten twice, recite alphabet twice, say mother and father's first name twice, little head movement, lengthy head movement). All video images are progressively scanned and saved under RAW format as TIFF files which are then organized under the naming structure PALI_SSSS_T_C_PP, where SSSS corresponds to the subject number running from 1 to 100 , $\mathrm{T}$ corresponds to the trial number, $\mathrm{C}$ stands for the lighting conditions, and PP stands for the protocol number. All files follow this taxonomy with each TIFF images having an additional 6 digits for the frame number. See Figure 6 for a set of ten sample images.

Eye coordinates for the entire database were generated using the Average of Synthetic Exact Filters (ASEF) algorithm [36] trained on the "illum" subset of the CMUPIE Database. In particular, the face images were first resizedto a 16:9 aspect ratio in order to correct the aspect

\footnotetext{
${ }^{3}$ We note that results achieved on the "illum" subset are comparable to those achieved on the "lights" subset. In the interest of space, they are not reported here.
} 

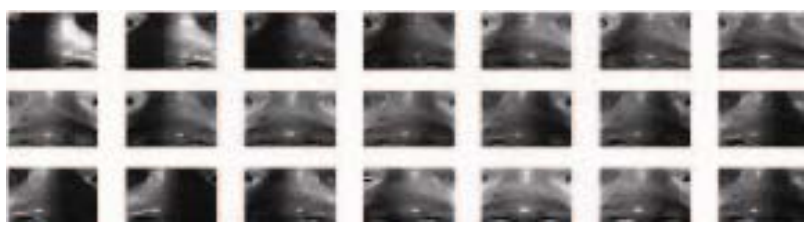

(a)
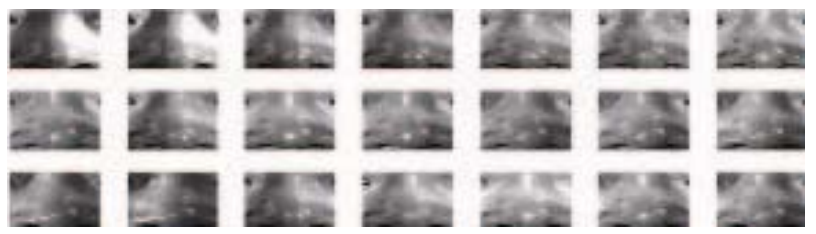

(b)

Figure 5. An illustration of the illumination variations for a fixed subject under the frontal pose in CMU-PIE Database. Left: “illum” subset; Right: "lights” subset.
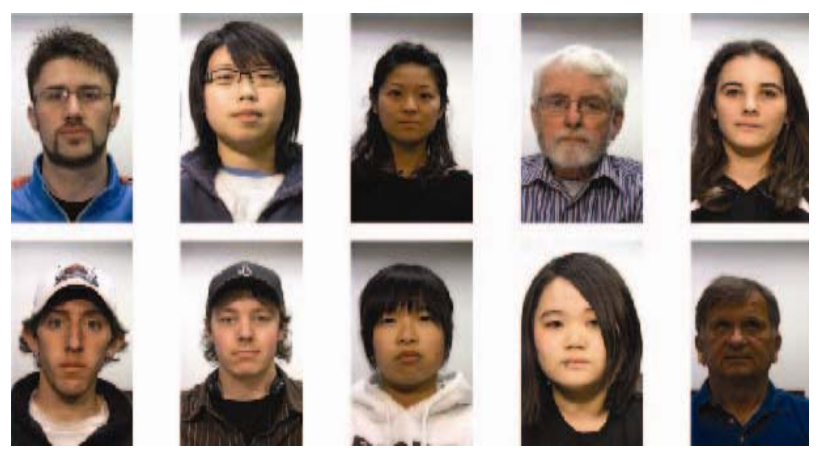

Figure 6. Sample images of CSU-PAL Database.

ratio of the images. The Open CV Cascade face detector [37] was used to detect faces and ASEF was used to locate the eyes. See Figure 7 for an illustrative example of the eye detector.

\subsection{Compressions of $n$}

For each of the 67 people in the CMU-PIE data set, we randomly select two sets of images of equal cardinality with disjoint illumination conditions. Since illumination spaces can be well-approximated by a 10-dimensionallinear subspaces [2,38], we randomly select two disjoint sets of size ten for the points in the probe and gallery. This process is repeated ten times producing a total of 670 probe points. Now, instead of the whole face image, selected feature patches are used. Note that the size of $n$ for each feature was chosen to include the maximum amount of the feature in the patch across all subjects in this set of experiments and the compression ratio is roughly $1 / 9$. The result of this experiment is given in Figure 8 along with the patch resolutions and the computational time required to calculate the distance between a single pair of probe and gallery points. Results for the baseline algorithm are also shown for comparison. Notice that while the proposed algorithm performs without
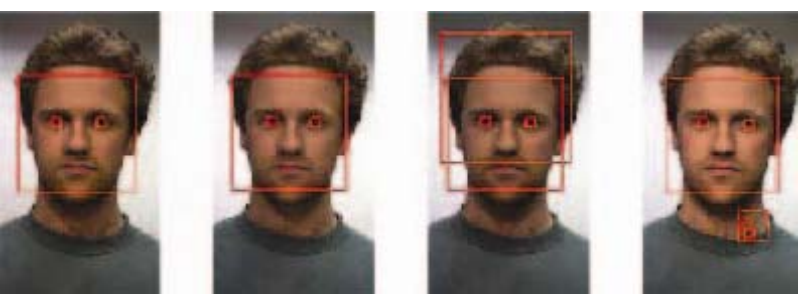

Figure 7. An illustration of the eye detector with ASEF algorithm [36].

error on this task, the baseline algorithm performs poorly and is computationally more expensive than classification on Grassmannians.

It is apparent from the results of the first experiment that when the cardinality of points in the gallery and probe is ten, the algorithm is able to separate all people in the data set using each of the selected patches without error. To further speed up the classification time and to see how sensitive the proposed algorithm is to the location of the feature patches, we repeat the experiment while reducing the patch resolution until the perfect recognition rates cease to exist. Figure 9 gives several extreme conditions where perfect recognition results continue to hold right before breaking. Notice that the baseline algorithm is extremely sensitive to patch resolutions and less efficient. For example, while using 87-pixel lip patches, the baseline algorithm attains an error rate of $6.82 \%$ and it takes 58 times longer to compute. The results here suggest that locally correlated feature patches consisting of an extremely small number of pixels provide sufficient information for recognition.

It is rather curious just how many pixels obtained through patch collapsing are necessary to retain sufficient information for recognition. To this end, we employ feature patches consisting of a random (but the same for each image) selection of 36 pixels. A set of ten different illuminations is used for both the gallery and probe. Hence, the data is represented as points on $G R(10,36)$. We find that the idiosyncratic nature of the patches persist in this extreme case. We performed the first experiment again, but now using randomly projected low-dimensional patches and still observed error-free identification for all people in the CMU-PIE Database. Perhaps surprisingly, a similar result is observed when we use a thin horizontal strip of just 33 pixels across the left eye.

The number " 36 " and " 33 " used here are more or less a result of the geometry on the Grassmannians, $G R(k, n)$. The number of principal angles equaling zero is bounded below by $2 k-n>0$. For example, two 2 -dimensional subspaces in $\mathbb{R}^{3}$ will necessarily intersect nontrivially generating at least one $(2 \times 2-3=1)$ principal angle equaling 0 and the extreme case scenario is that the two subspaces intersect completely generating two principal angles equaling 0 . On the other hand, two 2-dimensional 


\begin{tabular}{|c|c|c|c|c|c|c|c|}
\hline & & lip & nose & left eye & right eye & left cheek & right cheek \\
\hline & Resolution & $41 \times 59$ & $59 \times 39$ & $21 \times 41$ & $21 \times 41$ & $31 \times 37$ & $31 \times 37$ \\
\hline \multirow{2}{*}{ Grassmann Methed } & CPU time & 0.0037 & 0.0034 & 0.0011 & 0.0011 & 0.0014 & 0.0014 \\
\hline & FAR & 0 & 0 & 0 & 0 & 0 & 0 \\
\hline \multirow{2}{*}{ Baseline } & CPU time & 0.0254 & 0.0249 & 0.0187 & 0.0187 & 0.0198 & 0.0198 \\
\hline & FAR & 0.3008 & 1.2234 & 2.5690 & 4.8937 & 2.2388 & 4.8937 \\
\hline
\end{tabular}

Figure 8. FAR (in \%) for individual feature patches where 10 imagesare used to compute each point in the probe and gallery. On a $2.8 \mathrm{GHz}$ AMD Opteron processor, the CPU time is how long it takes to calculate the distance/similarity between a probe and a gallery point in seconds.

\begin{tabular}{|c|c|c|c|c|c|c|c|}
\hline & & lip & nose & left eye & right eye & left cheek & right cheek \\
\hline & Resolution & $3 \times 29$ & $35 \times 13$ & $21 \times 41$ & $21 \times 41$ & $31 \times 37$ & $31 \times 37$ \\
\hline Grassmann Methed & CPU time & $2.7 \times 10^{-4}$ & $6.3 \times 10^{-4}$ & 0.0011 & 0.0011 & 0.0014 & 0.0014 \\
\hline \multirow{2}{*}{ Baseline } & FAR & 6.8204 & 1.2121 & 3.1592 & 6.5762 & 4.1995 & 0.5812 \\
\hline & CPU time & 0.0158 & 0.0171 & 0.0187 & 0.0186 & 0.0199 & 0.0196 \\
\hline
\end{tabular}

Figure 9. Conditions for achieving zero FAR using proposed algorithm forindividual feature patches where ten images are used to compute each point in the probe and the gallery. FAR scores for baseline algorithm are also listed for comparison.

subspaces in $\mathbb{R}^{4}$ might not share a direction at all hence generating zero principal angle equaling 0 . Thus, if we restrict the subspace dimension to ten, i.e., $k=10$, then the ambient resolution dimension needs to be at least 21 in order to allow some wiggle room for non-intersecting behaviors. In this case, there are $\left(\begin{array}{l}21 \\ 10\end{array}\right)=21 \times 19 \times 17 \times$ $13 \times 11 \times 4$ ways to form a 10 -dimensional subspace in $\mathbb{R}^{21}$. This specification potentially allows a database of $3,879,876$ distinct subject to be uniquely represented as a point on $G R(10,21)$.

On the numerical side, it is helpful to know whether human error incurred during image registration has negative effects on the classification accuracy. To this end, we repeat the first experiment with varying registration and examine the classification error rates as a function of this variation. In the first experiment, feature patch images were captured by convolving with a fixed-position mask of 1's with size equaling the patch resolution. To generate images of varying registration, this mask is randomly shifted either horizontally or vertically one pixel at a time. Classification is repeated for every pixel shift up to 10 pixels using the new registered images to obtain error statistics. The lip and nose patches were the least sensitive to the particular perturbation of registration we utilized. The results imply that if a human operator registers the gallery patches in their own particular manner, then another human operator has about two pixels of freedom in registering the probe patches if spurious errors are to be avoided. Of course, expanding the data sets to include data that is poorly registered will likely improve this tolerance.

Thus far, we have illustrated a successful model for performing set-to-set classification of low resolution face images on a data set that is already "nice", i.e., images are registered and cropped. To introduce practicality, we consider a much noisier database, the CSU-PAL Database that is introduced in Section 4.1. In the interest of space, we consider only the left eye patches of size 181by-71 selected from the high-resolution video frames that are originally sized 1080 -by-1440 in this set of experiments. Similar results can be found on all other feature patches. Original images were first registered according to a procedure described in Section 4.1 and then cropped to the selected feature as shown in Figure 10. Since this paper concerns with only lighting variations, we consider the images under file structure PALI_SSSS_1_1_01.

For each of the 100 subjects in the CSU-PAL data set, 

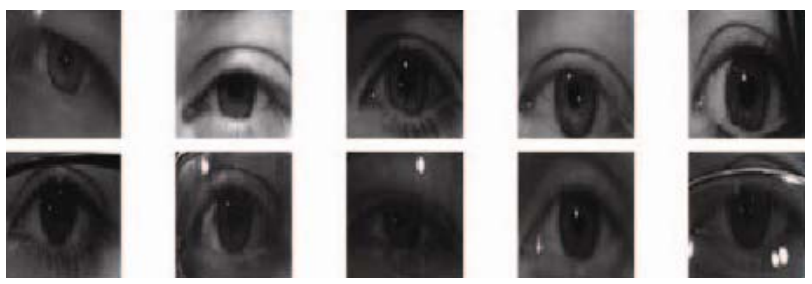

Figure 10. Sample images of left eye patches from the CSUPAL Database.

we randomly select two disjoint sets of size $p$ and $g$ for each subject in the probe and gallery, respectively, for comparison. In particular, we let the ordered pair, $(g, p)$, be $(10,10),(20,20),(50,50)$, and $(20,1)$. For all four experiments, this process of random selection is repeated ten times producing a total of 1000 probe points in each case. The error rate for each experiment is given in Table 1.

The results here show that perfect Grassmann separability is achieved with a 50-dimensional subject subspace representation. The fact that it requires many more images than the empirical dimension of illumination spaces is possibly due to the fact that the images are acquired under fewer constraints such as the appearance of eye glasses. While a near perfect separation result can be accomplished with a balanced 10-dimensional subspace representation, the proposed algorithm suffers from having an extremely small $k$ value. This result illuminates the next set of experiments in Section 4.3 where effects of compression of $k$ are examined.

\subsection{Compressions of $\boldsymbol{k}$}

In this experiment, we examine the effect of varying the number of images used in constructing the probe and gallery hence corresponding to compression in $k$. Often times, it is unrealistic to collect equal numbers of images at enrollment and during operation. Therefore, it is hard to avoid comparisons of sets of images of asymmetric sizes. In such cases, we would like to know the minimal number of images needed to represent a person while still achieving perfect separation. Figure 11 shows the classification error rates for each selected patch. The cardinality of the probe points increase from 1 to 20 while the cardinality of the gallery points simultaneously decrease from 20 to 1 . The illumination conditions for the probe are always disjoint from the conditions in the gallery. The plot suggests the performance of the algorithm is optimal when the cardinality of the probe and gallery points approach each other, i.e., a balanced comparison. For instance when considering the nose patch, using only one image per person in the probe and 20 images per person in the gallery yields an error rate of about $2.2 \%$, while the error rate diminishes to zero when using three images per person in the probe and 18 images per person
Table 1. FAR and standard deviation (in \%) for the left eye patches in CSU-PAL database. Cardinalities of the gallery and probe sets are given in the first row, respectively.

\begin{tabular}{ccccc}
\hline & $(10,10)$ & $(20,20)$ & $(50,50)$ & $(20,1)$ \\
\hline \multirow{2}{*}{ FAR } & $0.9 \pm 1.35$ & $0.1 \pm 0.03$ & 0 & $10 \pm 88.18$ \\
\hline
\end{tabular}

in the gallery.

In the worse case scenario, if it is only possible to collect a single image for each probe, then we would like to know the minimum number of images required for each person in the gallery in order to obtain perfect separation. For this set of experiments, we use a single image for each probe and let the cardinality of the gallery vary from 1 to 20. The classification error rates for each of the selected patches are given in Figure 12. For example, when using the lip feature, the algorithm performs perfectly using 16 images per person. However, when the cheek feature is selected, even the use of 20 images per person in the gallery could not force perfect recognition rates. Suggestively, certain features (e.g., nose, lip) provide more discriminatory information than others (e.g., cheeks) when classification is carried out via Grassmannians. This is perhaps not surprising seeing how human beings recognize novel faces. We often learn someone's face from facial features that are more geometrically curved. The curvature (in the general geometric sense) of the eye, nose, and lip regions are generally larger than that compared to the cheek. Therefore, these features are more pronounced in a 2-dimensional representation of the face, i.e., a digital image. Moreover, the Grassmann method is by nature a set-to-set method and one would expect decreased performance when the number of images per subject class is scarce. The subspace representation improves as the number of images approaches the intrinsic rank (or dimension) of the subject illumination spaces. This justifies the choice of $k=10$ in the experiments conducted in the previous subsection.

However, ideally we would like the dimensionality of the subject subspace representation to be the upper bound on the number of images needed to perform classification for two reasons - reduction in storage and computational cost. This is especially true for high-resolution video sequences. For example, if it costs 1 Megabyte (MB) to store an image, then a database of $10^{3}$ people each having $10^{3}$ images would take up 1 Terabyte (TB) of hard drive space. Although the disk space has become much cheaper to acquire, cost on the order of this magnitude is still undesirable. One way to reduce the cost for real-time storage space and number of comparisons is to exploit the explicit information afforded by an implicit representation; that is, a compact representation that captures the discriminatory characteristics exhibited in the image sets. To accomplish this task, we propose the use of 

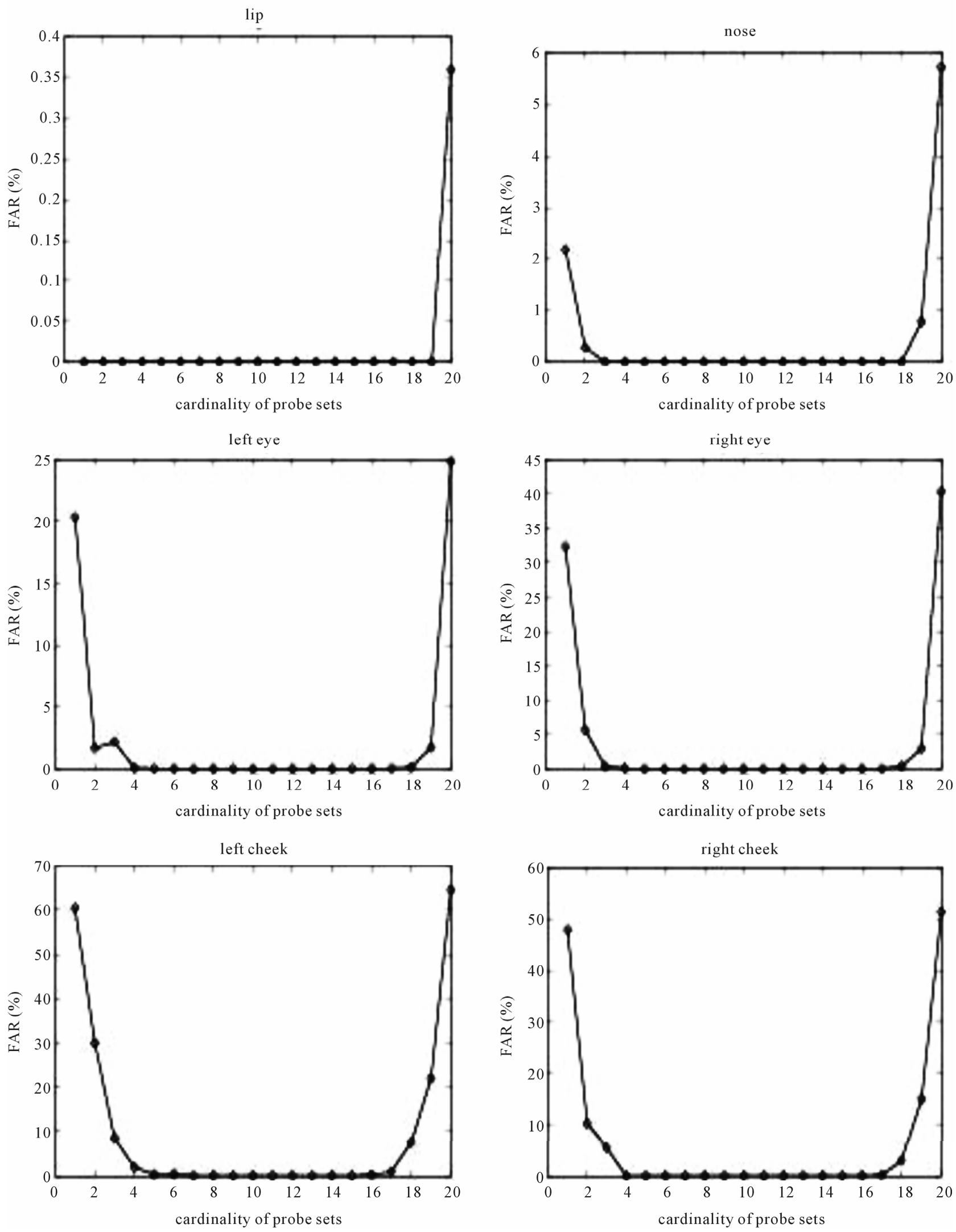

Figure 11. Classification error rates for each selected feature patch. The cardinality of points in the probe increases from 1 to 20 while the cardinality of points in the gallery simultaneously decreases from 20 to 1. 
lip
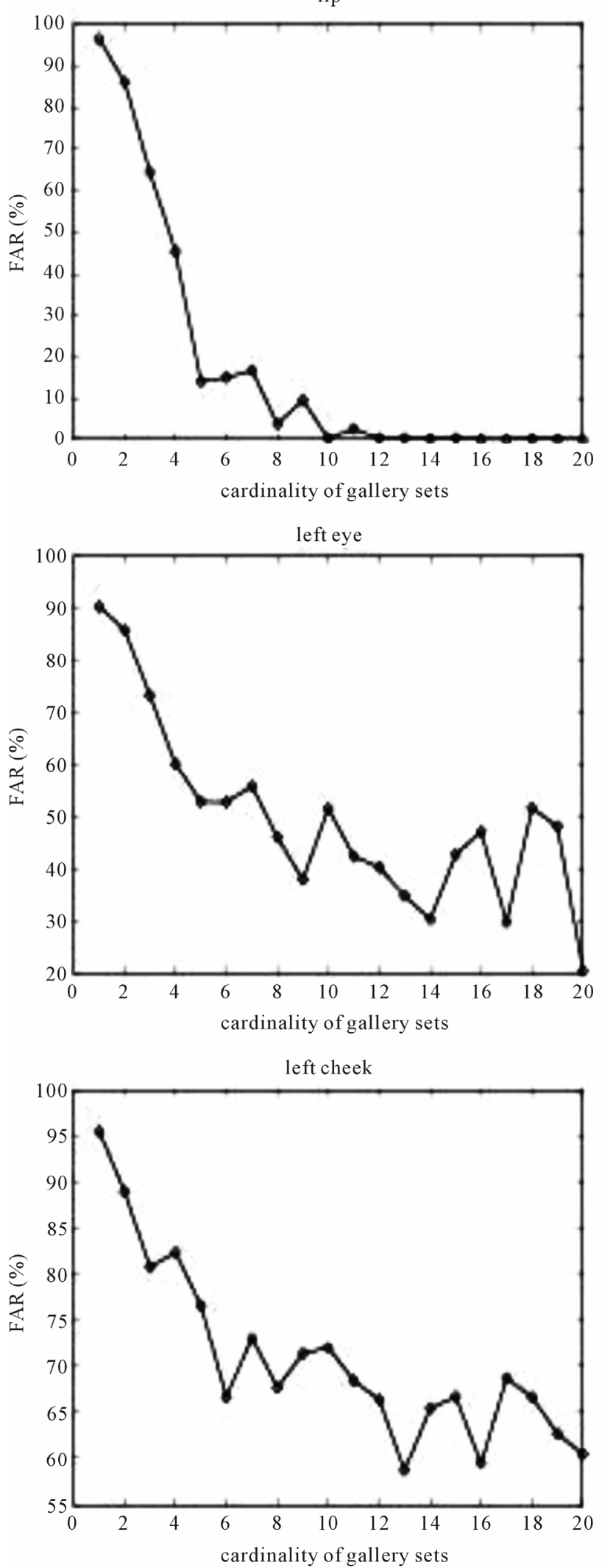
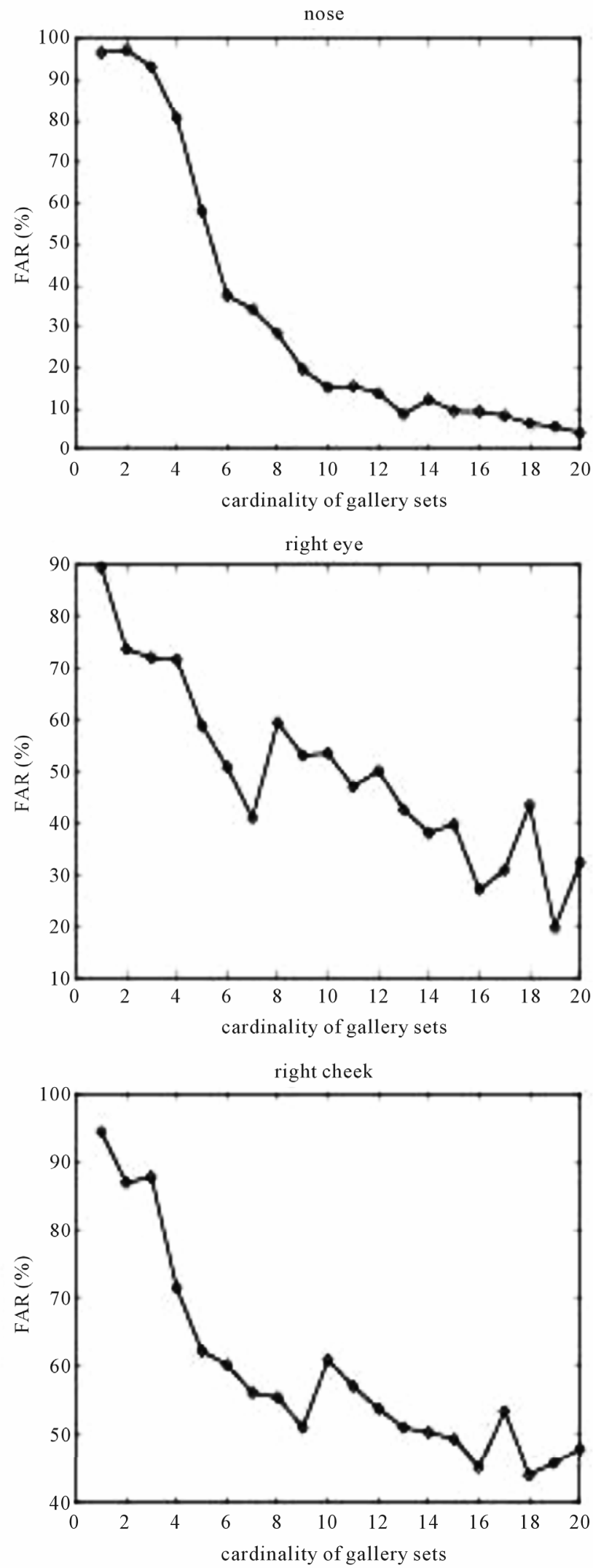

Figure 12. Classification error rates for each selected feature patch. The cardinality of the probe points is one while the cardinality of the gallery points ranges from 1 to 20. 
Karcher representation, as described in Algorithm 3, for image sets on the Grassmannians and illustrate its potential use for data compression with the following experiment on the CUM-PIE "lights" data set.

The way we validate whether this reduced representation can be used to replace the original image sets in the face recognition tasks is to compare the respective classification error rates. If an error-free classification result can be achieved on the original $G R(N, n)$, then for a Karcher representation to successfully compress the same discriminatory information, the same error-free result will need to be observed on $G R(k, n)$ for $1 \leq k \leq N$. The optimal result will be an error-free classification when the compression is at its maximum, i.e., when $k=1$. We will now describe the specific parameters implemented in this validating experiment.

Let $R^{(i)}$ be an $n \times N$ matrix that stores all $N$ gallery images of each subject class for $1 \leq i \leq P$. Denote $1 \leq k \leq$ $\lfloor N / 2\rfloor$ the dimensionality of the Karcher representation which will be explored throughout the experiment. Further let the cardinality of the probe sets be three for all subjects and store the images in $n \times 3$ matrices, $T^{(i)}$, $1 \leq i \leq P$. For a fixed $k$, Algorithm 3 is used to obtain a Karcher representation for each subject class, $\left\langle l^{(i)}\right\rangle_{i=1}^{P}$, where $P$ is the total number of subjects in the gallery. That is, each $\left\langle l^{(i)}\right\rangle$ resides in $G R(k, n)$ and can be represented by a $n \times k$ matrix, $K^{(i)}$. If we cast the classification architecture in a distance matrix with the minimal principal angle metric $D(\cdot, \cdot)$, then Karcher compression provides useful compact representation if $\tilde{d}_{i i}=\min _{1 \leq j \leq P} \tilde{d}_{i j}$, where $\tilde{d}_{i j}=D\left(K^{(i)}, T^{(j)}\right)$, given that $d_{i i}=\min _{1 \leq j \leq P} d_{i j}$ with $d_{i j}=D\left(R^{(i)}, T^{(j)}\right)$.

Let $N=16$, the $k$-dimensional Karcher representation resulted in an error-free classification for $k \geq 4$ on the lip patch of the "lights" data set $(n=41 \times 59=4919)$. In comparison, using four raw images per subject in the gallery resulted an average FAR of $30 \%$. In the extreme case, when only a single raw image is used, the classifier returns an error rate of nearly $90 \%$; while a 1 -dimensional Karcher representation returns a $1 \%$ error rate. Figure 13 tabulates the error rate as a function of the dimensionality of the Karcher representation as well as the cardinality of the gallery image set.

The fact that the compression of a raw point on $G R(16,41 \times 59)$ to a Karcher representation on $G R(4,41 \times 59)$, without diminishing performance, indicates the promise of Karcher compression in the context of classification of image sets via Grassmannians. On the contrary, when using $k$ raw images for each gallery point, the error rate never reaches zero for any $1 \leq k \leq 8$. The fact that using a 4-dimensional Karcher representation achieves a perfect recognition result while using four raw images in the gallery does not indicates that Karcher representations are able to pack useful discriminatory information in a more efficient manner. This technique can potentially be used to enable compact representations computed from video sequences or data sets where a large number of images is available for the gallery.

\section{Summary and Discussions}

In this paper, a geometric framework for the general classification problem with image sets is reviewed. The power of the method is due, in part, to the fact that the geometry and statistics of the Grassmann manifold are well-understood and provide useful tools for quantifying the relationships between patterns. We made precise how this geometric framework is translated in practical settings. We show by ways of experiments that the proposed Grassmann method is robust against resolution and dimensionality reduction which corresponds to compression in both $k$ and $n$ in $G R(k, n)$.

Although there might be other ways to accomplish either of these two compressions, we consider compression in $n$ induced by mathematical projections and compression in $k$ through the proposed Karcher representation. Empirical results collected on a public database, CMUPIE, and a private database, CSU-PAL, verify claimed success in employing a compressed representation through the use of Karcher mean and mathematical projections on the Grassmannian in set-to-set classification. These results are reported through an appropriate measure on the Grassmann manifold that coincides with a classifier metric termed false accept rate at a zero false reject rate, FAR for short.

The work presented here originated from our goal to push the Grassmann method to a breaking point. While

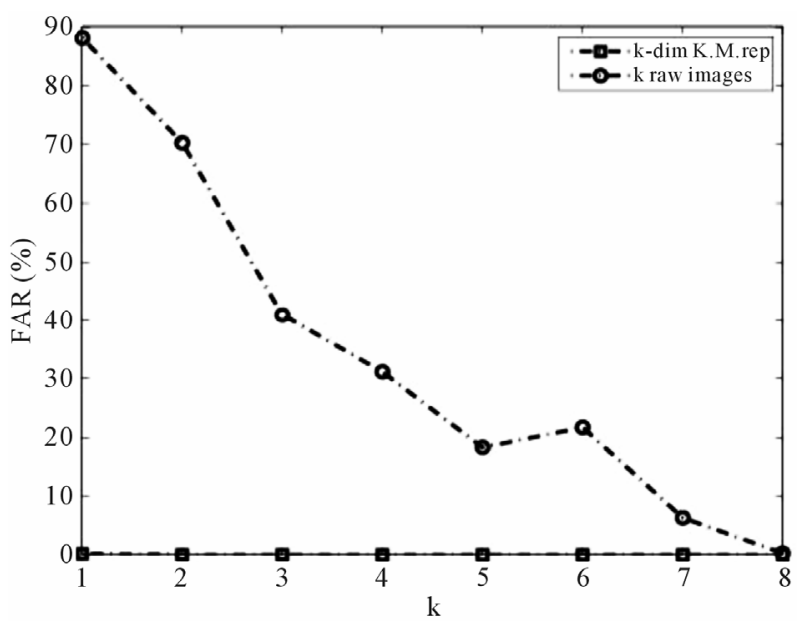

Figure 13. Error rate comparisons with $k$-dimensional Karcher representation and $k$ raw images for points in the gallery corresponding to lip patches. Three images are used to compute points in the probe. 
one may have many images in the gallery, often a very small portion of that set or the images is utilized. We are making identifications using illumination variations on a portion of the face. The results shown here provide an implementation blueprint in practice. Imagine a labeled gallery point where each image is of a person whose face is $95 \%$ occluded so that all you can see of the face is a fixed portion of the cheek. Now build a collection of such cheek image gallery sets for different people. The results in the paper suggest that you can determine from a probe cheek image set (if you again allow variation in illumination conditions) whether the probe cheek image matches another cheek image set in the gallery and which gallery set it matches. For this paper, we are imagining the scenario where a gallery has been built from the entire face. Now a probe person's images are collected within which "almost" their entire face is obscured. Our method requires we know which portion of the face is not obscured. Then we build a gallery of people's illumination spaces at this known portion of the face and make our comparisons to the probe on the Grassmannian.

An emphasis should be drawn to the fact that feature patches typically have a sufficiently small resolution, e.g., 50 - 100 pixels, such that the machinery of the Karcher mean is computationally tractable. A major contribution of the paper is that the Karcher mean computed on the Grassmannians can be used to compute a reduced representation of the gallery while still maintaining error-free recognition on the illumination patches. We speculate that this approach will pay increased dividends with larger data sets. We further remark that other parameter spaces such as Stiefel manifolds and flag manifolds also present opportunities for extensions of these ideas. Additionally, although we focus on illumination as the source of state variation, we remark that other variations in state, such as those obtained by multi-spectral cameras, also fit within this framework.

\section{Acknowledgements}

This work is partially supported by NSF DMS and DCCF grant MSPA-MCS 0434351 and DOD-USAF-Air Force FA-9550-08-1-0166. The authors gratefully thank the computing resources provided by the Pattern Analysis Laboratory at Colorado State University. The authors would also like to thank David Dreisigmeyer for helpful discussions concerning the Karcher Mean and David Bolme for providing the eye coordinates of the CSUPAL face imagery using ASEF.

\section{REFERENCES}

[1] A. Georghiades, P. Belhumeur and D. Kriegman, "From Few to Many: Illumination Cone Models for Face Recognition under Variable Lighting and Pose," IEEE Transac- tions on Pattern Analysis and Machine Intelligence, Vol. 23, No. 6, 2001, pp. 643-660. doi:10.1109/34.927464

[2] R. Basri and D. Jacobs, "Lambertian Reflectance and Linear Subspaces," IEEE Transactions on Pattern Analysis and Machine Intelligence, Vol. 25, No. 2, 2003, pp. 218-233. doi:10.1109/TPAMI.2003.1177153

[3] R. Gross, I. Matthews and S. Baker, "Fisher Light-Fields for Face Recognition across Pose and Illumination," Proceedings of the German Symposium on Pattern Recognition (DAGM), Vol. 2449, 2002, pp. 481-489.

[4] J.-M. Chang, J. Beveridge, B. Draper, M. Kirby, H. Kley and C. Peterson, "Illumination Face Spaces are Idiosyncratic," Proceedings of the International Conference on Image Processing \& Computer Vision, Vol. 2, 2006, pp. 390-396.

[5] O. Yamaguchi, K. Fukui and K. Maeda, "Face Recognition Using Temporal Image Sequence," Proceedings of the International Conference on Automatic Face and Gesture Recognition, 1998, pp. 318-323. doi:10.1109/AFGR.1998.670968

[6] K. Fukui and O. Yamaguchi, "Face Recognition Using Multi-Viewpoint Patterns for Robot Vision," Proceedings of the International Symposium of Robotics Research, Vol. 15, 2005, pp. 192-201.

[7] R. Wang, S. Shan, X. Chen and W. Gao, "ManifoldManifold Distance with Application to Face Recognition Based on Image Sets," Proceedings of the Computer Vision and Pattern Recognition Conference, 23-28 June 2008, pp. 1-8.

[8] A. Fitzgibbon and A. Zisserman, "Joint Manifold Distance: A New Approach to Appearance Based Clustering," Proceedings of the Computer Vision and Pattern Recognition Conference, Vol. 1, 2003, pp. 26-36.

[9] A. Srivastava, "A Bayesian Approach to Geometric Subspace Estimation," IEEE Transactions on Signal Processing, Vol. 48, No. 5, 2000, pp. 1390-1400. doi:10.1109/78.839985

[10] X. Liu, A. Srivastava and K. Gallivan, "Optimal Linear Representations of Images for Object Recognition," IEEE Transactions on Pattern Analysis and Machine Learning, Vol. 26, No. 5, 2004, pp. 662-666. doi:10.1109/TPAMI.2004.1273986

[11] G. Stewart and J.-G. Sun, "Matrix Perturbation Theory," Academic Press, New York, 1990.

[12] T. Kanade, "Picture Processing System by Computer Complex and Recognition of Human Faces," Ph.D. Thesis, Kyoto University, Kyoto, 1973.

[13] L. Wiskott, J.-M. Fellous, N. Krüger and C. Malsburg, "Face Recognition by Elastic Bunch Graph Matching," IEEE Transactions on Pattern Analysis and Machine Intelligence, Vol. 19, No. 7, 1997, pp. 775-779. doi:10.1109/34.598235

[14] R. Brunelli and T. Poggio, "Face Recognition: Feature Versus Templates," IEEE Transactions on Pattern Analysis and Machine Intelligence, Vol. 15, No. 10, 1993, pp. 1042-1052. doi:10.1109/34.254061

[15] Y. Kaya and K. Kobayasaki, "A Basic Study on Human Face Recognition," Proceedings of the Frontiers of Pat- 
tern Recognition, 1972, pp. 265-290.

[16] J. Harris, "Algebraic Geometry: A First Course," Springer, Berlin, 1992.

[17] A. Edelman, T. Arias and S. Smith, "The Geometry of Algorithms with Orthogonality Constraints," SIAM Journal on Matrix Analysis and Applications, Vol. 20, No. 2, 1999, pp. 303-353. doi:10.1137/S0895479895290954

[18] A. Björck and G. Golub, "Numerical Methods for Computing Angles between Linear Subspaces," Mathematics of Computation, Vol. 27, No. 123, 1973, pp. 579-594.

[19] A. Knyazev and M. Argentati, "Principal Angles between Subspaces in a Based Scalar Product: Algorithms and Perturbation Estimates," SIAM Journal of Scientific Computing, Vol. 23, No. 6, 2002, pp. 2008-2040. doi:10.1137/S1064827500377332

[20] J. Beveridge, B. Draper, J.-M. Chang, M. Kirby, H. Kley and C. Peterson, "Principal Angles Separate Subject Illumination Spaces in YDB and CMU-PIE," IEEE Transactions on Pattern Analysis and Machine Intelligence, Vol. 31, No. 2, 2009, pp. 351-356. doi:10.1109/TPAMI.2008.200

[21] M. Nishiyama, O. Yamaguchi and K. Fukui, "Face Recognition with the Multiple Constrained Mutual Subspace Method," Proceedings of the International Conference on Audio- and Video-Based Biometric Person Authentication, 2005, pp. 71-80.

[22] M. Nishiyama, M. Yuasa, T. Shibata, T. Wakasugi, T. Kawahara and O. Yamaguchi, "Recognizing Faces of Moving People by Hierarchical Image-Set Matching," Proceedings of the Computer Vision and Pattern Recognition Conference, 2007, pp. 1-8.

[23] Q.-S. Sun, Z.-D. Liu, P.-A. Heng and D.-S. Xia, "A Theorem on the Generalized Canonical Projective Vectors," Pattern Recognition, Vol. 38, No. 3, 2005, pp. 449452. doi:10.1016/i.patcog.2004.08.009

[24] Q.-S. Sun, P.-A. Heng, Z. Jin and D.-S. Xia, "Face Recognition Based on Generalized Canonical Correlation Analysis," Advances in Intelligent Computing, Vol. 3645, 2005, pp. 958-967.

[25] J.-M. Chang, M. Kirby and C. Peterson, "Set-to-Set Face Recognition under Variations in Pose and Illumination," Proceedings of the Biometrics Symposium, Baltimore, 11-13 September 2007, pp. 1-6. doi:10.1016/j.patrec.2009.06.002

[26] T. Wang and P. Shi, "Kernal Grassmannian Distances and Discriminate Analysis for Face Recognition from Image Sets," Pattern Recognition Letters, Vol. 30, 2009, pp. 1161-1165. doi:10.1016/j.patrec.2009.06.002

[27] J. Hamm and D. Lee, "Grassmann Discriminate Analysis: a Unifying View on Subspace-Based Learning," Pro- ceedings of the 25th International Conference on Machine Learning, Vol. 307, 2008, pp. 376-383.

[28] M. Harandi, C. Sanderson, S. Shirazi and B. Lovell, "Graph Embedding Discriminant Analysis on Grassmannian Manifolds for Improved Image Set Matching," Proceedings of the Compute Vision and Pattern Recognition Conference, 20-25 June 2011, pp. 2705-2712.

[29] H. Cevikalp and B. Triggs, "Face Recognition Based on Image Sets," Proceedings of the Compute Vision and Pattern Recognition Conference, 13-18 June 2010, pp. $2567-$ 2573.

[30] A. Mansfield and J. Wayman, "Best Practices in Testing and Reporting of Biometric Devices: Version 2.01," Tech. Rep. NPL Report CMSC 14/02, Centre for Mathematics and Scientific Computing, National Physical Laboratory, UK, 2002.

[31] H. Karcher, "Riemannian Center of Mass and Mollifier Smoothing," Communications on Pure and Applied Mathematics, Vol. 30, 1977, pp. 509-541. doi:10.1002/cpa.3160300502

[32] W. Kendall, "Probability, Convexity and Harmonic Maps with Small Image I: Uniqueness and Fine Existence," Proceedings of the London Mathematical Society, Vol. 61, 1990, pp. 371-406. doi:10.1112/plms/s3-61.2.371

[33] E. Begelfor and M. Werman, "Affine Invariance Revisited," Proceedings of the Computer Vision and Pattern Recognition Conference, Vol. 2, 2006, pp. 2087-2094.

[34] J. Manton, “A Globally Convergent Numerical Algorithm for Computing the Center of Mass on Compact Lie groups," Proceedings of the International Conference on Control, Automation, Robotics and Vision, Vol. 3, 2004, pp. 2211-2216.

[35] T. Sim, S. Baker and M. Bsat, "The CMU Pose, Illumination, and Expression Database," IEEE Transactions on Pattern Analysis and Machine Intelligence, Vol. 25, No. 12, 2003, pp. 1615-1618. doi:10.1109/TPAMI.2003.1251154

[36] D. Bolme, B. Draper and J. Beveridge, "Average of Synthetic Exact Filters," Proceedings of the Computer Vision and Pattern Recognition, 20-25 June 2009, pp. 20152112.

[37] P. Viola and M. Jones, "Robust Real-Time Face Detection," International Journal of Computer Vision, Vol. 57, No. 2, 2004, pp. 137-154. doi:10.1023/B:VISI.0000013087.49260.fb

[38] P. Belhumeur and D. Kriegman, "What Is the Set of Images of an Object under All Possible Lighting Conditions," International Journal of Computer Vision, Vol. 28, No. 3, 1998, pp. 245-260. doi:10.1023/A:1008005721484 